\title{
ASPECTOS DE SALUD EN ADULTOS MAYORES DE 80 ANOS \\ DE EDAD QUE VIVEN INDEPENDIENTEMENTE EN LA \\ COMUNIDAD: UNA PERSPECTIVA AUSTRALIANA
}

Angélica Orb ${ }^{1}$

Orb A. Aspectos de salud en adultos mayores de 80 anos de edad que viven independientemente en la comunidad: una perspectiva australiana. Rev Latino-am Enfermagem 2004 julho-agosto; 12(4):589-96.

Este estudio cualitativo describe los factores que influenciaron las practicas de salud de un grupo de 14 adultos mayores que gozaban de un buen estado de salud y vivían en forma independiente en Perth, Australia Occidental. En entrevistas con los participantes, las prácticas de salud fueron examinadas. Cinco temas emergieron del estudio. Estos temas fueron el tiempo pasado, una vida activa, la alimentación, el envejecimiento y la seguridad económica y la satisfacción personal. Este estudio examina la vida de los adultos mayores y los problemas asociados con la edad avanzada.

DESCRIPTORES: anciano; actividades recreativas; salud del anciano

\section{HEALTH ASPECTS IN ADULTS OVER 80 WITH INDEPENDENT COMMUNITY LIFE: AN AUSTRALIAN \\ PERSPECTIVE}

This qualitative study describes the factors that influence the health care practices of 14 elderly persors living independently in Perth, Western Australia. Through in-depth interviews with the participants, the health care practices people were explored. Five distinct themes emerged from the data. These themes were the past time, an active life, nutrition, getting old, economical security and personal satisfaction. This study provides an insight into the life of older adults and the problems associated with ageing.

DESCRIPTORS: aged; leisure activities; adult health

\section{ASPECTOS DE SAÚDE EM ADULTOS ACIMA DE 80 ANOS QUE VIVEM DE MANEIRA INDEPENDENTE NA COMUNIDADE: UMA PERSPECTIVA AUSTRALIANA}

Este estudo qualitativo descreve os fatores que influenciaram as práticas de saúde de um grupo de 14 idosos com boas condições de saúde que viviam de maneira independente em Perth, Australia Ocidental. Em entrevistas com os participantes, as práticas de saúde foram examinadas. Cinco temas surgiram do estudo. Estes temas foram o tempo passado, uma vida ativa, a alimentação, o envelhecimento, a segurança econômica e a satisfação pessoal. Esta pesquisa analiza a vida dos idosos e os problemas relacionados com a idade avançada.

Descritores: idoso; atividades de lazer; saúdo do idoso

\footnotetext{
${ }^{1}$ RN, PhD, MACE, Alpha Chi, Senior Lecturer, School of Nursing \& Midwifery, Research Fellow, Freemasons Center for Research into Aged Care Services, Curtin University of Technology, e-mail: A. Orb@curtin.edu.au
} 


\section{INTRODUCCIÓN}

$\boldsymbol{H}_{\text {oy en día el numero de adultos mayores en el }}$ mundo esta creciendo rápidamente. Lo que es mas aun, el numero de personas mayores de 80 anos crecerá proporcionalmente. Estos cambios demográficos se deben en parte a los avances en las ciencias de la salud, especialmente en lo relacionado con el control de enfermedades cardiovasculares ${ }^{(1)}$. Con el aumento significativo de adultos mayores de 65 anos, lógicamente aumentaran las enfermedades mentales ${ }^{(2)}$.

El crecimiento demográfico afectara en gran medida a los países desarrollados pero también tendrá repercusiones en los países en vías de desarrollo. Consecuentemente, habrá un cambio en la estructura social, como así mismo en el área de la salud y la economía $a^{(1)}$. Australia con un total de $18,495,115$ millones de habitantes ${ }^{(3)}$, tiene aproximadamente 1.9 millones de adultos mayores sobre 65; se espera que para el ano 2031 este numero subirá a 5.2 millones. En otras palabras habrá un adulto mayor por cada ocho personas, en cambio en países como Suecia hay uno por cada cinco. Esta cifra es cercana a las predicciones para el ano 2020 en que la población sobre 65 será de uno cada cuatro ${ }^{(4)}$. Por otra parte, el numero de personas sobre 85 anos crecerá 10 veces mas, esperándose que el numero de mujeres sobre 85 aumentara en un $3 \%^{(1)}$. McCallum y Geiselhart ${ }^{(1)}$ consideran que en Australia en el ano 2030 habrán 100 mujeres por cada 52 hombres mayores de 85 anos.

Heidrick y D'Amico ${ }^{(5)}$ indican que hay una falta de estudios que describan las actividades físicas y mentales de los adultos sobre los 80 anos $^{(5)}$. Otros como Wondolowski y Davis nos informan que al preguntarles a un grupo de 108 adultos mayores entre 80 y 100 anos, que significaba ser saludable, estos respondieron que sentirse saludable significaba tener energía y vitalidad; que esta vitalidad los hacia moverse y sentirse con fuerzas ${ }^{(6)}$. Estar saludable les permitía sentirse satisfechos en el sentido que podían completar una tarea y recordar los aspectos positivos del pasado. En Australia y especialmente en Australia Occidental el numero de estudios relacionados con las percepciones de los adultos mayores y el proceso de envejecimiento es limitado, especialmente en aquellos sobre los 80 anos de edad.

Hasta ahora la tendencia en las sociedades desarrolladas ha sido presentar al adulto mayor como una persona enferma que eventualmente terminara sus últimos días en un asilo de ancianos. Pocas veces se celebran los alcances y logros de este grupo, se habla mas de la patología del adulto mayor, con énfasis en problemas como demencia y movilidad. Sin embargo, en Australia las estadísticas confirman que el $95 \%$ of los adultos mayores de 65 anos vive en forma independiente no dependiendo de los servicios de salud ${ }^{(7)}$. Es importante recordar que los adultos mayores que hoy tienen mas de 80 anos son el producto de una sociedad que sufrió dos guerras, la Depresión de los anos 30 y cambios sociales y tecnológicos de gran envergadura.

Como profesionales de la salud interesados en el bienestar de nuestros adultos mayores, tenemos la responsabilidad de conocer de cerca las experiencias que han vivido este grupo y preguntarnos si estamos preparados para ofrecerles los servicios que sus necesidades requieren. ¿Cuánto sabemos de las necesidades de salud? ¿Cuales son sus motivaciones? Sin una base de información es imposible planear las necesidades futuras de este grupo. Orb et al. consideran que la edad mas comúnmente usada para clasificar a una persona como adulto mayor son los 65 anos $^{(8)}$. Otros como Kozier et al. clasifican a los adultos mayores en tres grupos: a) 64-74 anos, adultos mayores jóvenes, b) 75-84, adultos mayores medianos; y 3) sobre 85 , estos son clasificados como adultos mayores-mayores ${ }^{(9)}$. Este ultimo grupo se considera el más vulnerable y por lo tanto el más dependiente de los servicios de salud.

\section{OBJETIVO}

El objetivo principal de este estudio fue preguntarles a un grupo de adultos mayores de 80 anos, cuales eran los factores que influyeron en las practicas de salud que les permitían mantenerse saludables.

\section{COLECCIÓN DE DATOS}

Los datos se obtuvieron a través de entrevistas y notas tomadas durante y después de las entrevistas. La mayoría de los participantes entrevistados vivían independientemente en departamentos propios bajo la administración de la Iglesia Anglicana. La administración general de estas residencias fue contactada y se solicito autorización para distribuir una invitación a aquellos adultos 
mayores de 80 anos que estarían interesados en participar en este estudio. Once participantes fueron seleccionados de este grupo residencial. Otros dos fueron seleccionados de un condominio privado y un tercero vivía en una casa residencial. Todas estas residencias están ubicadas en la zona metropolitana de Perth, en el estado de Australia Occidental. Los centros comerciales y otras facilidades, como el uso de correo, banco o centros de salud quedan a una distancia de 4 o 5 cuadras de estas residencias.

El proyecto fue aprobado por el comité de ética de la Curtin University of Technology. La muestra total fue de 14 participantes. El criterio de selección se baso en la edad, 80 anos o más, encontrarse en buen estado de salud y ser capaz de hablar ingles claramente. Las entrevistas se realizaron en el hogar de cada participante. Antes de comenzar los participantes fueron informados del objetivo del estudio, y de los derechos de cada uno de ellos, se puso énfasis en la confidencialidad de la información. Cada entrevista duro entre 60 y 90 minutos. Se considero que saturación se había alcanzado cuando los participantes comenzaron a repetir la misma información ${ }^{(10)}$.

\section{CARACTERÍSTICAS DE LOS PARTICIPANTES}

Las edades de los participantes en este estudio fluctuaron entre los 80 y 92 anos. Nueve eran mujeres y cinco hombres. De las nueve mujeres, siete eran viudas, una casada y una divorciada. Cuatro de los cinco hombres eran casados y uno viudo. Tres vivían con sus esposas y el otro la visitaba regularmente en una residencia para pacientes con Alzheimer. De los 14 participantes, 11 vivían solos.

\section{MÉTODO DE ANÁLISIS}

Las entrevistas fueron grabadas y se transcribieron palabra por palabra. Las notas tomadas durante y después de las entrevistas, fueron incluidas como parte del contenido del estudio. Todos las entrevistas fueron escuchadas y cotejadas con las transcripciones, luego cada entrevista se leyó varias veces con el fin de entender e interpretar lo que los participantes expresaban. La información fue codificada y luego que ciertos temas comenzaron a emerger, cada uno se analizo en el contexto total de la información obtenida. Con frecuencia se hicieron preguntas a la información obtenida con el fin de clarificar y comprender el fenómeno que se estudiaba. Los resultados del análisis se discutieron y verificaron con un grupo de participantes.

\section{RESULTADOS}

A pesar de que los participantes reconocieron que sufrían achaques y dolores, todos se consideraban saludables. Muchos de ellos atribuyeron este estado de salud al hecho de haber tenido una vida ordenada, una niñez y una vida de adulto activa. Además una alimentación balanceada. Para otros el buen estado de salud se relacionaba con poder movilizarse sin ayuda y al mismo tiempo tener una buena calidad de vida. Como se sabe, con frecuencia las traducciones hacen perder el mensaje que se desea enviar, por esta razón en este articulo se han incluido en ingles las propias palabras de los participantes.

El tiempo pasado

Para la mayoría de los participantes en este estudio, los días de la niñez y juventud tuvieron mucha importancia sobre el estado de salud en que se encontraban. La mayoría de ellos expresaron que su niñez y juventud estuvo llena de actividades deportivas como natación, tenis, golf, ciclismo y caminatas, a pesar de que no tenían mucha afluencia económica. Ellos consideraron que aquellas actividades desarrolladas en la niñez y juventud fueron factores decisivos en el estado de salud actual.

He jugado football y críquet y también fui un buen jugador de tenis. Jugué tenis, golf y bolos.

I've done a bit of football and criket, and I was quite a good tennis player. (I played) tennis and golf and bowls. Participante 3

Por ejemplo, algunos participantes dijeron:

Bueno jugué tenis. No muy bien pero intente jugar. Como vivíamos en el campo y no había nada que hacer, hacíamos picnic. También caminábamos bastante, además hacíamos picnic los domingos estos eran organizados por el colegio.

Well I played tennis. Not very well but I mean to played. And we lived in the country. I mean there was nothing else to do. We did a lot of walking and pinics and Sunday Schools picnics. Participante 8 
¡Oh! entonces caminaba hacia el monumento de Forrest en la esquina de Terrace y la calle Barrack. Por supuesto, no teníamos permiso para caminar a través de los jardines, allí había un hermoso árbol de jacarandá, todavía esta allí.

Oh then I would walk up to the Forrest Memorial on the corner of The Terrace and Barrack Street and we were not allow to walk thought the gardens, there was a beautiful jacaranda tree there, and it's still there. Participante 6

Los días de la niñez y juventud fueron días difíciles para este grupo de adultos mayores, todos habían sufrido los efectos de la Depresión of los anos 30 Las dificultades económicas no les permitían participar en actividades que significaran muchos gastos debiendo contentarse con actividades que no demandaran mayor desembolso. A pesar de las dificultades económicas, estas al parecer no los afecto emocionalmente y es así como uno de los participantes dijo que a sugerencia de su padre, se convirtió en un buen caminante:

Mi padre me dijo que caminar era mas barato. No se necesitaba uniforme, solamente los zapatos. Esto era en el peor tiempo de la Depresión de los 30. Creo que las cosas eran más difíciles. Mucho más difíciles que ahora. Yo era un niño, no sabia nada, esto fue difícil.

Because my father said it was cheap. No uniform and no, only the shoes. This was in the worst part of the 30's Depression. So I mean things were tigh. Much tighter than they are now. I was a child, I did not know it was difficult. Participante 10

\section{Una vida activa}

De acuerdo con los participantes la memoria de una niñez saludable, a pesar de las dificultades vividas durante la Depresión, les dejo un rastro en la forma como ellos perciben la salud. Para mantenerse saludables ellos consideraron importante tener una vida lo mas activa posible. Esto lo consiguen desarrollando pequeñas tareas en casa, trabando en el jardín o desarrollando hobbys. Por ejemplo, una persona de 87 anos dijo:

Cuando viví en mi otra casa trabaje mucho en el jardín, en ese tiempo no caminaba mucho. Pero ahora, si el tiempo esta bueno me agrada caminar todos los días. Ahora tengo un problema con mis piernas, porque tengo una estrechez de las arterias sobre las rodillas.

When I lived in the house I did a lot of gardening and I didn't do much walking then. But here, if the weather is nice I like to go for a walk everyday. I have a problem now with my legs because I have narrowing of the arteries above the knees. Participante 4
Otros de los participantes comentaron que debido a problemas de movilidad llevaban una vida sedentaria pero trataban de mantenerse lo mas activo posible, a pesar de la edad y los problemas asociados con el envejecimiento. Por ejemplo un participante dijo:

No me siento como uno de 92 para nada. No sé, me siento normal. Excepto por mi movilidad, siempre deseo caminar.

I don't feel like a 92 at all. I don't know, I just feel normal. Except for the area of mobility but I want to walk around. Participant

La mayoría considero que era importante mantenerse intelectualmente activos. Algunos se refirieron a la importancia de mantenerse al día a través de los diarios y la televisión. También se refirieron a la lectura, las palabras cruzadas, a los juegos de naipes y reuniones sociales pero además estaban contentos con su propia compañía.

Una persona de 89 anos comento:

Una pareja de amigos viene a verme cada 15 días, vienen todos los viernes nos tomamos un trago, un café con queque. Generalmente invito a mi amigo de arriba con su señora, los cuales me agradan mucho....ellos vienen aquí con mi otro amigo que tengo aquí, nos visitamos cada 10 días y nos tomamos unos tragos, prácticamente aquí a todos nos gusta tomamos unos tragos, estamos prácticamente alcohólicos, ¿entiende? ....siempre estamos visitándonos para tomarnos un trago.

One couple come here every fortnight, they come here every other Friday and have a drink, coffee and cake and I usually invite my friend and her husband from upstairs I have grown to like them very much...they all come down here and my other friends that I have here we go to each others units every ten days and have drinks, we are practically alcoholic here you know... were always popping into each other for drinks. Participante 9

Otros comentaron:

Los domingos en la noche nos tomamos un trago. Ud. sabe ese tipo de entretención. No soy el tipo de persona que le gusta tomar. Nunca me aburro con mi propia compañía y no siempre tengo que estar llamando a otros para que me hagan compañía.

On Sunday night we have a drink together. You know it's a sort of thing. I' $m$ not a sort of person that's, no I don't get bored with my own company. I don't have to be always calling on people and things. I don't do much of that at all. Participante 2

Escucho música diariamente. Nunca me aburro con mi propia compañía.

(I listen to) some music every day. I never get bored with my own company. Participante 3 
Alimentación

Los participantes de este estudio consideraron esencial tener una dieta balanceada. Para ellos una buena alimentación ha jugado un rol importante en su estado de salud. Se consideraba una buena alimentación la inclusión de frutas y verduras frescas en la dieta diaria, además de la reducción de carnes de vacuno y grasas. Otro aspecto que consideraron importante fueron las comidas frecuentes y en pequeñas cantidades. Por ejemplo uno de los participantes indico:

Todo lo que como es bajo en grasa. No como carne, no me gusta, como pollo, pescado, fruta y verdura y como dije siempre una dieta baja en grasa.

Everything I eat is low fat. I don't eat meat at all, I don't like it, chicken, fish, fruit vegetables and as I say a low fat diet always. Participante 9

Otros participantes indicaron que no estaban acostumbrados a comer comida chatarra y aquellos que venían de una zona rural indicaron que estaban acostumbrados a comer comida casera.

Me crié en el campo y todo se cultivaba en casa o localmente. Nada de comida chatarra y ocasionalmente comíamos pescado.

I was brough up in the country and everthing was sort of home grown or locally grown. Nothing was fast food and occasionally you'd get some fish. Participante 8

La misma participante agrego que actualmente comía mas frecuentemente pero en porciones más pequeñas:

Yo como. Como mucha fruta y verdura, digo, como carne, pero no mucho. No como grandes comidas ni tampoco como entre las comidas, pero me encanta el chocolate. Claro, tengo que tener cuidado porque subo de peso.

I eat. I eat a lot of fruit and vegetables and not, I mean I eat meat, but not a great big lot. I don't eat great meals and I don't eat in between meals but I love chocolate. But I have to be careful because I put on weight. Participante 8

Envejecimiento

Los participantes consideraron que se encontraban en buen estado de salud pero hablaron de algunas dificultades que los afectaban por el hecho de ser adultos mayores. Varios aspectos emergieron del análisis de la información, entre estos estaba el proceso de envejecimiento, la perdida de confianza en si mismos los temores con que se enfrentaban por el hecho de ser de edad avanzada lo cual los limitaba en la vida social. El tema Envejecimiento se subdividio en dos sub temas:

- Perdida de la confianza en sí mismos. Estos participantes indicaron que al llegar a la vejez se sentían físicamente diferentes comparado con los anos de juventud. Estaban más lentos, la capacidad física se había reducido y estaban más olvidadizos. De todas maneras estaban muy conscientes de que a medida que avanzaban en edad había ciertos aspectos de la vida que ya no eran como antes. Para algunos este proceso biológico los había hecho perder la confianza en si mismo y sentirse más vulnerables, especialmente con las caídas. Otros sentían tener reducido los niveles de energía para realizar tareas físicas. Una forma de paliar esta situación era tomar las cosas con calma y darse tiempo.

Un participante de 92 anos indico que se cansaba fácilmente pero tomaba su tiempo para realizar sus actividades. A pesar de todo tenia temores de caerse.

Si, me canso fácilmente, si....me siento. Continua diciendo: Estuve un poquito cansado todo el día, el día antes de que Ud. viniera, donde are incapaz de hacer nada. Ahora tengo una niña (se refiere a una persona del servicio domestico) que me ayuda a ducharme porque no tengo mucha estabilidad y no quiero caerme en la ducha.

Yes, I tire easily, yes... I sit down. I 've even had a day. The day before you arrived I was bit knackered all day...... I get the girl in now to give me a shower now because l'm not good enough on my feet and I don't want to fall down in the shower. Participante 3

- Limitaciones en la vida social. Participantes comentaron que la vejez los había hecho reducir sus actividades sociales. Había varias razones que redujeron la vida social. Primero que nada, en algunos casos las limitaciones físicas habían reducido la agilidad, se sentían más vulnerables y in cierta medida habían perdido su autonomía. Otra de las razones era molestias menores como los siguientes casos. Un participante de 84 anos indico que la sordera le limitaba su vida social. Otra persona dijo que por sufrir de diverticulosis y diarreas, esto también le limitaba la vida social. No se atrevía a salir porque en cualesquier momento podría necesitar con urgencia ir al baño.

Algunos reconocieron que les era imposible llevar el ritmo que la vida de cuidad les imponía. El ritmo acelerado de la vida moderna con mucho movimiento vehicular fue uno de los aspectos que ha repercutido en la vida de los adultos mayores. Por ejemplo, manejar en 
carreteras de alta velocidad, tener que cambiarse de pista rápidamente o tomar las líneas del transito correspondientes al lugar donde deseaban llegar fueron algunos de los factores que decidieron a algunos participantes dejar de manejar, a pesar de que otros aun lo hacían. Un participante de 92 anos dijo:

Mi movilidad es mi punto flaco. Recientemente recibí mi ultimo informe medico que me permite moverme. Todavía voy de compras y todavía manejo mi automóvil. Es muy conveniente ir de compras.

My mobility is my lowest point and just recently got the last report back to enable me to move around and that. Like going shopping and that. I can still drive my motor car. That's handy to go up to shops. Participante 3

Otros en cambio indicaron que dejaron de conducir debido a la presión familiar. La falta de transporte propio les limitaba su movilidad haciéndolos depender del transporte publico. Lo cual significaba una gran dificultad. Algunos de los participantes expresaron no tener confianza en el transporte publico, prefiriendo el use de taxis, lo que consecuentemente aumentaba sus gastos.

\section{Seguridad económica y satisfacción personal}

Todos los participantes en este estudio indicaron que debido a sus entradas reducidas tenían cuidado con sus gastos. Algunos de ellos se habían hecho su propia jubilación mientras otros recibían la jubilación del gobierno. Algunas parejas consideraron que la ayuda económica que recibían del gobierno era suficiente. No así aquellos que vivían solos.

Le digo que, no es muy divertido vivir de mis propios fondos de retiro. No tengo derecho a la jubilación del gobierno, no tengo ayuda de ninguna clase.

I tell you what, it's not much fun living on a superannuation because you don't get any pension help, you don't get any help of any sort. Participante 7

De todas maneras, a pesar de que los participantes no tenían mucha afluencia de dinero, si estaban contentos con su vida pasada y presente. Algunos estaban agradecidos de cada día que transcurría. Muchos vivían con los buenos recuerdos del pasado y contentos con su propia compañía. El hecho de tener una familia que los recordara y los visitara también era importante para ellos.

Medito y rezo. Me toma 20 minutos rezar por todos mis hijos, por todos los nietos y por mi bisnieto y por aquellos que nacieron a fines de ano, y esto completa realmente mi día.

I meditate and pray. It takes me 20 minutes for all the children, all my grandchildren, and all my great grand child, and the ones that have been born later on this year. And that fills my day really. Participante 6

Un participante de 87 anos agrego que un buen matrimonio también era parte de la felicidad.

Tuve una buena esposa y eso es otra cosa que supongo hacen que uno sea feliz en su matrimonio.

I had a good wife and that's another thing I suppose, that you are happy in your marriage. Participante 5

\section{DISCUSIÓN}

Es evidente que los resultados de este estudio nos permiten tener una percepción mas clara de los factores que han influido en la mantención del estado de salud de estos adultos mayores. Tanto el tiempo pasado como el presente parece tener relevancia cuando hablan de la salud. La mantención de un buen estado de salud descrita por los participantes revela, no solo la importancia de la actividad física sino también la importancia de una buena dieta alimentaría. Pascussi y Loving mencionan la importancia de una dieta en base a frutas y verduras en su estudio con adultos mayores de 100 anos $^{(11)}$. Por otro lado, al parecer las actividades deportivas llevadas a cabo durante la niñez y la juventud dejaron una huella significativa en el actual estado de salud de estos participantes. A pesar de que la mayoría de ellos estaban conscientes de la importancia de mantenerse activos, algunos, sobretodo aquellos que tenían mas de 87 anos, tenían dificultades para hacerlo. Siendo al temor a las caídas uno de los mayores problemas. Este aspecto esta ampliamente documentado en la literatura ${ }^{(12)}$ y se la ha dado mucha importancia en el área de la prevención. En relación con la falta de fuerzas y energía, el servicio de salud en el ámbito comunitario les ofrece un servicio de mantencion del hogar, domestico y transporte a todas los adultos mayores $^{(1)}$ que requieren ayuda, el cual puede ser de una o dos veces por semana dependiendo del nivel de dependencia del adulto mayor. Por otro lado, las municipalidades en el estado de Australia Occidental ofrecen una gama de actividades físicas para personas sobre 50 anos. Entre estas actividades están las caminatas en grupos, ejercicios en el agua, o ejercicios de bajo impacto. Estas actividades son promovidas a un bajo costo 
entre los adultos mayores. Hartshorn et al. reportan los beneficios de los ejercicios controlados sobre un grupo de adultos mayores que atendieron a cuatro sesiones de 50 minutos de ejercicios terapéuticos ${ }^{(13)}$. Estos autores indican que la función motora mejoro, especialmente la firmeza para caminar, también mejoro la fuerza de las piernas y disminuyo el dolor de ellas.

A pesar de que la mayoría de estos adultos mayores habían reducido las actividades físicas, para ellos mantenerse mentalmente activos era parte de su bienestar personal. En este sentido los servicios comunitario australianos también les dan oportunidades para asistir a paseos, picnic $u$ otras actividades sociales ${ }^{(7)}$. Jones y Jones; Hogstel y Kashka confirman la importancia de la actividad física en los adultos mayores, la cual aumenta el tono muscular y mejora la agilidad ${ }^{(14-15)}$.

La mayoría de los participantes en este estudio consideraron que era necesario aceptar el proceso natural de envejecimiento. Ellos hablaron de los achaques y dolores que presentaban, el tiempo que les demoraba desarrollar ciertas actividades, los temores que tenían debido a los posibles accidentes que podrían enfrentar y lo que es mas, reconocer que la vida moderna iba más rápida que lo que eran capaces de enfrentar. Como se sabe los cambios en el sistema nervioso aparecen con la edad, y los reflejos son mas lentos, así mismo el ritmo acelerado de la vida moderna puede ser una de las grandes barreras para las personas mayores de 80 anos, si la familia, y los servicios de salud y comunitario no les proveen con las facilidades necesarias. El deterioro neurológico que ocurre con la edad ${ }^{(16)}$ hace que estos adultos mayores se sientan vulnerables frente a los cambios acelerados de la sociedad en que viven.

Russell después de haber observado la vida de un grupo de adultos mayores en un club para adultos mayores en un suburbio de Sydney, llego a la conclusión

\section{REFERENCES}

1. McCallum J, Geisehart K. Australia's new aged. Issues for young and old. Sydney: Allen \& Unwin; 1996.

2. Jeste DV, Alexopoules GS, Bartels SJ, Cummunig JL, Gallo JJ, Gottlich GL, et al. Consensus statement on the crisis in geriatric mental health. Arch Gen Psychiatry 1999; 56:848-53. 3. Australian Bureau of Statistics. Census of Population and Housing: Selected Social and Housing Characteristics. Australia: Australian Bureau of Statistics; 2002. Cat. No. 2015.0.

4. Dickenson-Hazard N. Living longer. Albatross or opportunity. Reflections Nurs Leadership 2001; 27(1):6. que la cantidad de dinero que este grupo disponía no estaba en directa proporción con la calidad de vida pero si, la soledad era un problema ${ }^{(17)}$. En el presente estudio los participantes indicaron que estaban contentos con su propia compañía, por lo tanto la soledad no era un problema pero si consideraron que el aspecto económico si era una desventaja. En este caso, tal vez, el hecho de vivir en una comunidad donde podían recurrir a sus amigos cuando lo deseaban, disminuye la soledad que frecuentemente se menciona en relación a los adultos mayores. Si, en cambio Minichiello et al. se refieren a las dificultades económicas de algunos participantes en su estudio de 18 adultos mayores que vivían en Victoria y Nueva Gales del Sur ${ }^{(18)}$. Igualmente, en este estudio que se presenta aquí, los adultos mayores indicaron que eran cuidadosos con sus gastos y que la situación económica no les permitía ciertos lujos pero si estaban contentos con su vida ${ }^{(19)}$.

\section{CONCLUSIÓN}

Los resultados de este estudio coinciden con lo señalado en la literatura, especialmente con los estudios en adultos mayores sobre 100 anos en Canadá y USA. Además de presentarnos un cuadro de la realidad de las percepciones de salud de estos adultos mayores, sirve de referencia y base de discusión para que las autoridades de salud y municipales puedan tomar medidas que faciliten el bienestar de estos ciudadanos. Dada la importancia que tiene el aspecto económico en el bienestar del adulto mayor, seria necesario repetir este estudio en una sociedad menos afluente y ver como este aspecto gravita en las practicas de salud en aquellas sociedades y al mismo tiempo identificar las medidas preventivas que podrían promoverse para que el adulto mayor tenga una mejor calidad de vida.

5. Heidrick SM., D'Amico D. Physical and mental relationships in the very old. J Commun Health Nurs 1993; 10(1):11-21.

6. Wondolowki C, Davis K. The lived experienced of health in the oldest old: A phenomenological study. Nurs Sci Q 1990; 4(3):113-8

7. Davis JD. Older Australians. A positive view of ageing. Sydney: W.B. Saunders: Bailliere Tindall; 1994.

8. Orb A, Davis P, Wynaden D, Davey M. A best practice model in psychogeriatric care. Aust N Z J Ment Health Nurs 2001:10:10-9.

9. Kozier B, Erb G, Blais K. Concepts and issues in nursing practice. $2^{\text {nd }}$ ed. Redwood City (CA): Addison-Wesley; 1992. 
10. Morse JM. Strategies in sampling. In: Morse JM, editor. Qualitative nursing research. A dialogue (revised ed.). Newbury Park: Sage Publication;1989. p. 127-45.

11. Pascucci MA, Loving GL. Ingredients of an old and health life: A centenarian perspective. J Holistic Nurs 1997; 15(2):199213.

12. Flicker L. Evidence-based health care in residential facilities for older people. Conference Proceedings. Improving quality of life in residential facilities for older persons. 25 May 2000. Perth: Positive Ageing Foundation; 2000.

13. Hartshorn K, Delage J, Field T, Olds L. Senior citizens benefits from movement therapy. J Bodywork and Movement Ther 2002; 6(1):55-8.

14. Jones JM, Jones KD. Promoting physical activity in the senior years. J Gerontol Nurs 1997; 23(7):40-8.

15. Hogstel MO, Kashka M. Staying healthy after 85. Geriatr Nurs 1989; 16-8.

16. Ebersole P, Hess P. Towards healthy aging. Human needs and nursing response. St Louis: C.V. Mosby; 1990.

17. Russell C. The aging experience. In: Davis JD. Older Australians. A positive view of ageing. Sydney: W.B. Saunders: Bailliere Tindall; 1994. p.76-9.

18. Minichiello V, Browne J, Kendig H. Perceptions and consequences of ageism: Views of older people. Ageing Soc 2000; 20:253-8.

19. Orb A, Davey M. The secret of long life. Perth: Curtin University of Technology; 2001. 